\title{
Low-frequency Mosaicism of Trisomy 14, Missed by Array CGH
}

\author{
Cha Gon Lee', Jun-No Yun², Sang-Jin Park ${ }^{3}$ and Young Bae Sohn²*
}

${ }^{1}$ Department of Pediatrics, Eulji General Hospital, Seoul, Korea; ${ }^{2}$ Department of Medical Genetics, Ajou University School of Medicine, Suwon, Korea; ${ }^{3} \mathrm{MG}$ MED, Inc., Seoul, Korea

\begin{abstract}
Mosaic trisomy 14 syndrome is a well-known but unusual chromosomal abnormality with a distinct and recognizable phenotype. Array comparative genomic hybridization $(\mathrm{CGH})$ analysis has recently become a widely used method for detecting DNA copy number changes, in place of traditional karyotype analysis. However, the array CGH shows a limitation for detecting the low-level mosaicism. Here, we report the detailed clinical and cytogenetic findings of patient with low-frequency mosaic trisomy 14, initially considered normal based on usual cut-off levels of array $\mathrm{CGH}$, but confirmed by G-banding karyotyping. Our patient had global developmental delay, short stature, congenital heart disease, craniofacial dysmorphic features, and dark skin patches over her whole body. Estimated mosaicism proportion was $23.3 \%$ by G-banding karyotyping and $18.0 \%$ by array $\mathrm{CGH}$.
\end{abstract}

Key words: Array CGH, Mosaicism, Trisomy 14, Developmental delay, Intellectual disability

\section{Introduction}

Chromosome mosaicism is defined as the presence of one or more cell populations with different genotypes in some of the body's cells in the same individual. Chromosomal mosaicism has been implicated as a leading genetic cause of human prenatal death, early prenatal brain development, congenital malformations, and many human diseases. ${ }^{1-3)}$ Several renowned mosaic chromosome syndromes include genetic disease such as Pallister-Killian syndrome (tetrasomy 12p mosaicism [PKS, OMIM 601803]), Hypermelanosis of Ito (HMI, OMIM 300337) and milder versions of known diseases (e.g., mosaic Down syndrome and mosaic Klinefelter syndrome). ${ }^{4)}$ Mosaic trisomy 14 syndrome is a well-known but unusual chromosomal abnormality with a distinct and recognizable phenotype. ${ }^{5-10)}$ The clinical features include retarded growth before and after birth, mental retardation, developmental delay, cardiac anomalies, minor anomalies of genitalia in boys, abnormal skin pigmentation and dysmorphic features. ${ }^{3)}$

Array comparative genomic hybridization (CGH) analysis has recently become a widely used method for detecting DNA copy number changes, in place of traditional karyotype analysis. In particular, an international consensus statement has now recommended array CGH as a first-line test in patients with unexplained developmental delay, intellectual disability and dysmorphisms. ${ }^{11)}$ However, the ability of array CGH is limited in case of low-level mosaicism. ${ }^{12}$ Here, we report the detailed clinical and cytogenetic findings of the first case in Korea to show lowfrequency mosaicism of trisomy 14 , diagnosed by array CGH analysis supplemented with karyotyping.

Received: 1 May 2013, Revised: 4 June 2013, Accepted: 4 June 2013, Published: 30 June 2013

${ }^{*}$ Corresponding author: Young Bae Sohn, M.D.,Ph.D.

Department of Medical Genetics, Ajou University School of Medicine, San 5, Wonchun-dong, Yeongtong-gu, Suwon, 443-721, Korea

Tel: +82-31-219-4522, Fax: +82-31-219-4521, Email: ybsohn@ajou.ac.kr

(c) This is an open-access article distributed under the terms of the Creative Commons Attribution Non-Commercial License (http://creativecommons.org/licenses/by-nc/3.0/) which permits unrestricted non-commercial use, distribution, and reproduction in any medium, provided the original work is properly cited.

(c) Copyright 2013 by the Korean Society of Medical Genetics

www.e-kjgm.org 


\section{Case Report}

\section{Clinical Findings}

A 6.4-year-old girl was referred to the clinical genetics clinic for evaluation of short stature, dysmorphic features and developmental delay. She was born at 41 weeks of gestational age by spontaneous vaginal delivery after an unremarkable pregnancy. She was the first child of non-consanguineous Korean parents: a 28-year-old healthy mother and a 29-year-old healthy father. Her birth weight was 3,220 g $\left[-0.05\right.$, standard deviation $\left.(S D)^{13}\right]$. She had a healthy younger brother. The family history was negative for stillbirths, infant deaths, birth defects, developmental delays and genetic disorders.

At birth, she showed clubfeet. Her parents reported that she had poor sucking power, feeding difficulty, and poor weight gain during her first year of life. She underwent ligation of the patent ductusarteriosus (PDA) at 8 months of age.

She showed apparent global development delay from early infancy. She held her head at 4-5 months, walked independently at 27 months, and used one word with meaning at 15 months. She exhibited apparent language disability: she made short sentences with two words at the age of 6.4 years. There was no history of seizure.

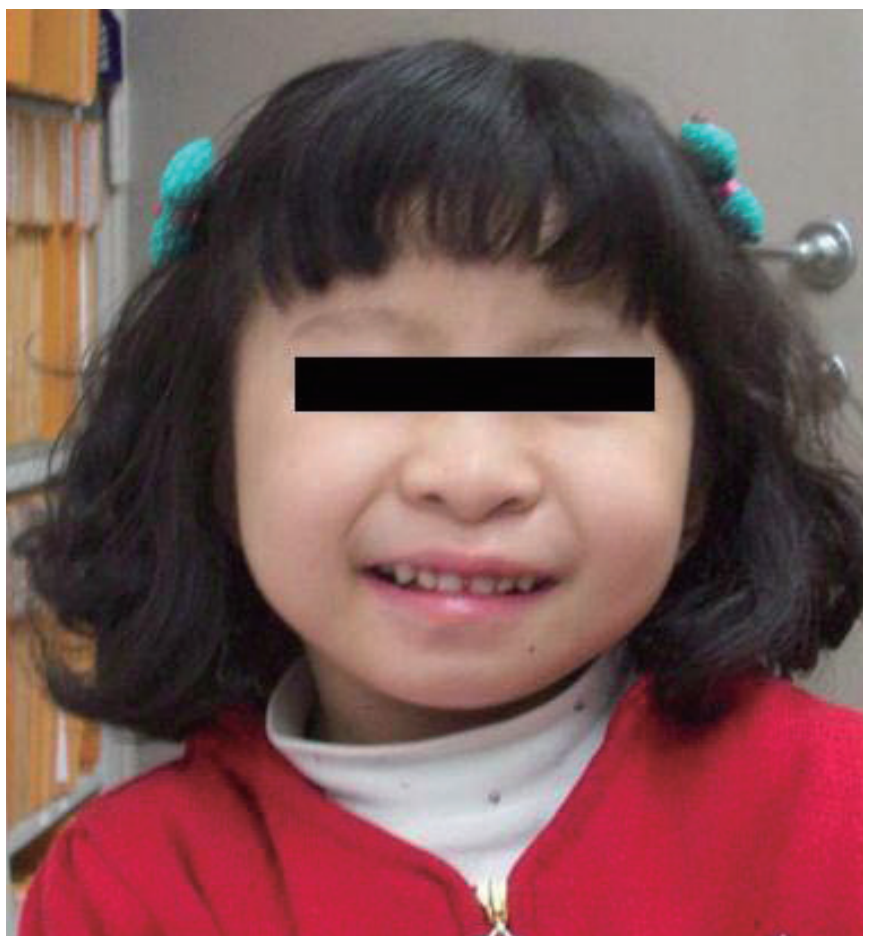

Fig. 1. Patient shows round shaped face, broad upturned nose, depressed nasal bridge, large mouth, widely spaced teeth and short neck.
When she initially visited our clinic as a 6.4-year-old, she exhibited shortstature: her height was $101.4 \mathrm{~cm}$ (-3.4SD), body weightwas $18.2 \mathrm{~kg}(-1.2, \mathrm{SD})$, body mass index (BMI) was $17.7 \mathrm{~kg} / \mathrm{m}^{2}$ (-1.6SD), and head circumference was $51.1 \mathrm{~cm}(+0.2 \mathrm{SD})$. On examination, she had round shaped face, broad upturned nose, depressed nasal bridge, large mouth, widely spaced teeth, and short neck (Fig. 1). She presented multiple hyperpigmented patches on her whole body, especially on the upper and lower extremities. Musculoskeletal and neurological examinations were normal.

A metabolic screening test by tandem mass spectrometry (MS) was normal. All other laboratory tests, including complete blood count, chemistry panel, thyroid function test, and urinalysis were normal. Radiologic exam revealed no evidence of abnormalities. Insulin like growth factor (IGF-1) was $714 \mathrm{ng} / \mathrm{ml}$ (normal range for age, $48.5-214 \mathrm{ng} / \mathrm{ml}$ ) and insulin like growth factor binding protein 3 (IGFBP-3) was 3.23 mg/L (normal range for age, 1.5-3.4 mg/L). Bone age was retarded for the chronologic age: estimated bone age was $4.5-5$ years at her age of 6.4 years.

\section{Array CGH Analysis and Chromosome analysis}

We conducted a whole genome array comparative genomic hybridization (array CGH) using commercially available array CGH slides (MACArray Karyo 1440 BAC-chip, Macrogen, Seoul, Korea). The array CGH chip data were analyzed using a chromofluor image analysis system (Macrogen, Seoul, Korea). Test to reference ratios were automatically determined for each sample and the means reported as a $\log _{2}$ Test/Reference (T/R ratio) signal ratio graph. An average $\log _{2} T / R$ signal ratio value more than +0.250 (2.5 SD) was considered as a chromosome number gain, whereas an average $\log _{2} \mathrm{~T} / \mathrm{R}$ signal ratio value less than -0.250 (2.5SD) was classified as a chromosome number loss.

Array $\mathrm{CGH}$ analysis indicated that all $\log _{2} \mathrm{~T} / \mathrm{R}$ ratios were within $\pm 2.5 \mathrm{SD}$ (Fig. 2). The spot of chromosome 14 was also within $\log _{2} \mathrm{~T} /$ $R$ ratio 0.250 , but it was slightly increased to 0.124 when compared with the other chromosomes (Fig. 2). We conducted G band standard karyotyping, considering a possibility of low-level copy number changes in chromosome 14. Chromosome analysis was conducted on $30 \mathrm{G}$-banded metaphase cells from peripheral blood T-lymphocytes according to routine cytogenetic protocols. The G-banding analysis revealed mosaic trisomy 14 (Fig. 3): 47, XX +14 [7]/46, XX [23]. Thus, the percent aneuploidy on karyotyping was $23.3 \%$. 
We calculated the percentage of mosaicism as recommended by Cheung et al ${ }^{14)}$ The formula for the calculation is as below: $\delta$ is the estimated fold change from $\log _{2} T / R$. The estimated mixing proportion by array CGH was 18.0\%.

$$
\begin{aligned}
& \alpha=\frac{|(\delta-1)|}{0.5} \\
& \%=100 \times \alpha \\
& \delta=\text { estimated fold change from } \log _{2} \mathrm{~T} / \mathrm{R}
\end{aligned}
$$

\section{Ethical approval}

Ethical approval was provided by the institutional review board of Ajou University Medical Center, Suwon, Korea (AJIRB-MEDMDB-12-136).

\section{Discussion}

Chromosome 14 spans approximately 109 million base pairs and represents between 3 and 3.5\% of the total DNA in cells. Chromosome 14 likely contains between 700 and 1,300 genes. Many genetic conditions are related to changes in particular genes on this chromosome. Copy number changes of chromosome 14 have also been implicated as a cause of developmental and health problems. ${ }^{14}$

${ }^{15)}$ Mosaicism of trisomy 14 is one of the well-established causes of a genetic syndrome with variable phenotype; this syndrome has recurrent and cardinal features. Many reports indicate that most patients with mosaicism of trisomy 14 have growth retardation and some degree of developmental delay. ${ }^{3,9}{ }^{155}$ Approximately $60-90 \%$ patients have congenital heart defects, such as atrial or ventricular septal defects. ${ }^{2,3,5,9,15)}$ Common craniofacial dysmorphisms are prominent forehead, broad/short/or upturned nose, dysplastic and/or apparently low set ears, large mouth, micrognathia, and short neck. ${ }^{2,3,59}$ Another frequent finding is abnormal skin-pigmentation. ${ }^{2,3,5,9,15)}$ Mosaic trisomy 14 syndrome is reported with a diverse proportion of trisomic cell lines, ranging from 4 to $70 \%{ }^{2,3)}$ Previous reports indicate that clinical phenotypes have no correlation with the percentage of trisomic cells. ${ }^{3)}$ Despite her relatively low-level mosaicism (23\% in karyotyping), the clinical features of the our patient were consistent with previous studies, and included global developmental delay, short stature, congenital heart disease including PDA, craniofacial dysmorphic features, and darkskin patches over her whole body. Previously, only one case of Korean patient with mosaic trisomy 14 was reported; 17-month-old girl who had 44\% mosaic trisomy based on karyotyping. ${ }^{15)}$ Our case showed similar
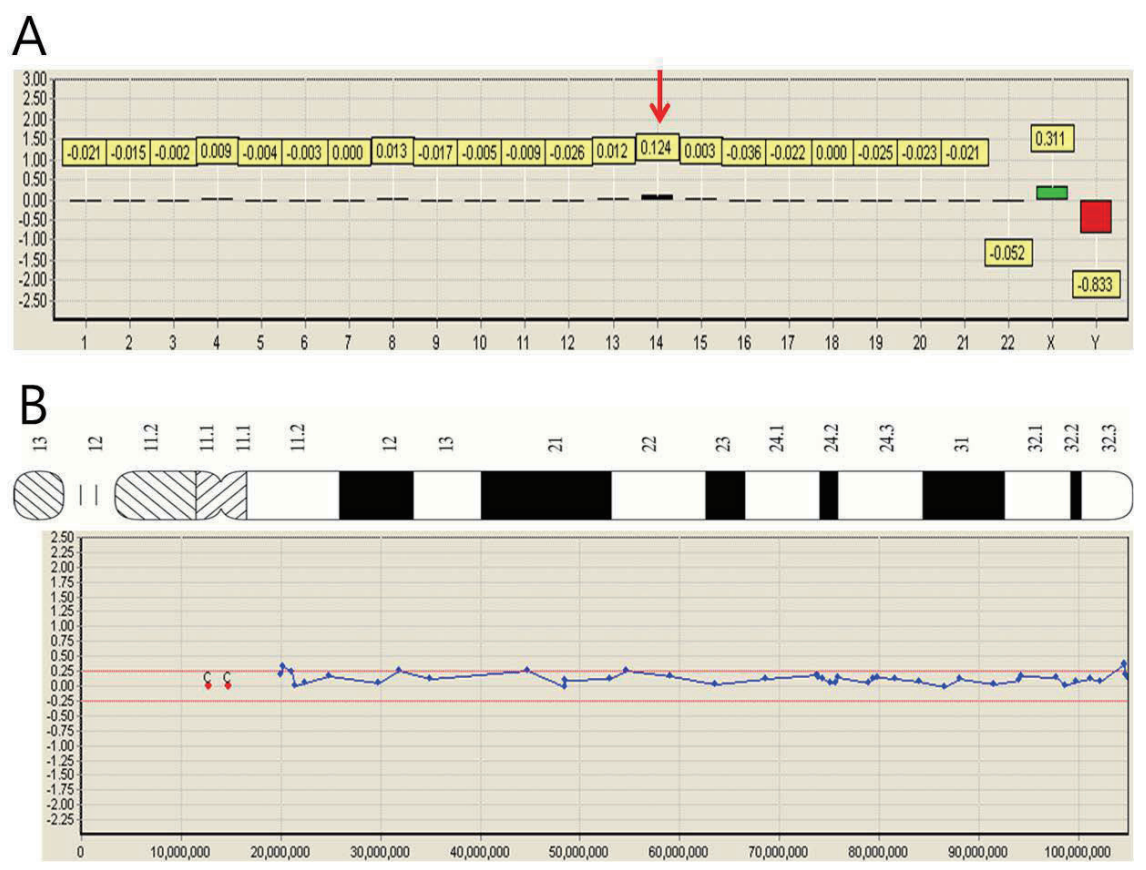

Fig. 2. (A) The X-axis represents chromosome number (1-22, $X, Y)$ and the $Y$-axis represents the $\log _{2} T / R$ signal ratio value. $\log _{2} T / R$ values of autosomes were all within \pm 0.250 . The $\log _{2} T / R$ signal ratio of chromosome 14 was slightly increased to 0.124 compared to the other autosomes. This is suggestive of chromosome 14 mosaicism. (B) $\log _{2} T / R$ was within 0.250 , indicating that copy number gains of chromosome 14 were substantially increased, but not high enough for consideration as an abnormal result. 
clinical features compared with previous Korean case, including broad nasal bridge, anteverted nose, and wide lip, abnormal skin hyperpigmentation, failure to thrive, and delayed development.

Mosaicism aneuploidy arises from mitosis or meiosis. Co-occurrence of mosaic trisomy and uniparental disomy (UPD) was reported in some case. ${ }^{16,17)}$ Up to date, the UPD has been described in association with mosaic trisomy 14 in only one patient with supernumerary marker chromosome $\left.14 .{ }^{18}\right)$ Therefore, short tandem repeat (STR) marker study to rule out the possibility of coexist with UPD would be necessary in our case.

As we know, array CGH has several advantages over G-banded karyotyping. Array $\mathrm{CGH}$ is a non-culture cell based technique and does not need dividing cells. Therefore, turnaround time can be reduced to usually a few hours. Array $\mathrm{CGH}$ also shows higher resolution than G-banded karyotyping and enables the detection of submicroscopic chromosomal segment gains and losses. ${ }^{111}$ However, array $\mathrm{CGH}$ cannot detect balanced structural rearrangements such as translocation and inversion, changes in DNA sequences, or gains and losses in regions of the genome not covered by the array $\mathrm{CGH}^{1{ }^{11)}}$ Low proportion mosaicism is also not detected by array $\mathrm{CGH}$. The ability of array CGH to detect low-level mosaicism is not precisely known. Based on a literature review, array CGH is unable to detect a frequency of abnormal cell mosaicism below 20-30\%. ${ }^{12)}$ However, some reportsare published thatarray CGH is more feasible to diagnose low level mosaicism than traditional karyotyping. Ballif et al. ${ }^{19)}$ analyzed over 3,600 clinical cases. They found out 18 cases of mosaicism. Fourteen cases of 18 were not known at initial analysis by karyotyping and were detected by only array $\mathrm{CGH}$. In all cases, confirmatory fluorescence in situ hybridization (FISH) analyses were performed on phytohemagglutinin (PHA)-stimulated cells and on un-stimulated cultures. They documented that that array CGH can detect the mosaicism as low as $10-20 \%$ and find even the missed by previous karyotyping. They also suggested that array $\mathrm{CGH}$ based on genomic DNA extracted from

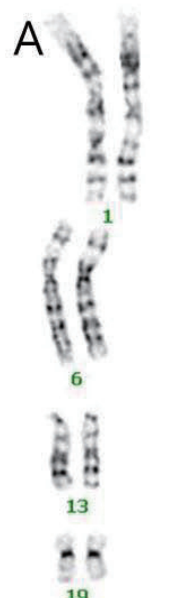

19
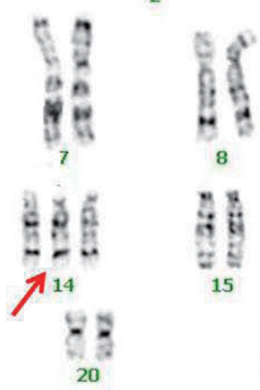

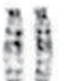

15
B

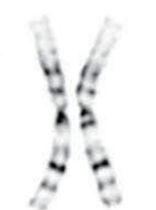

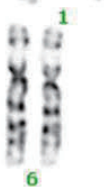

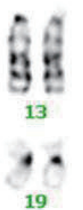

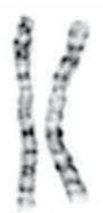

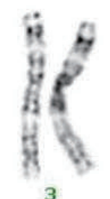

3
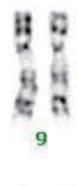
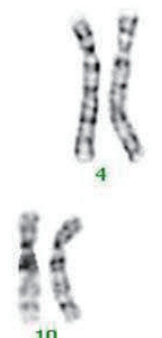

10

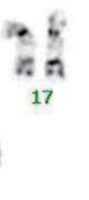

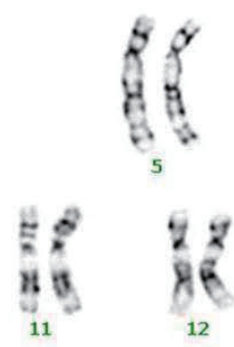

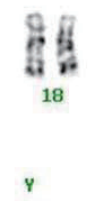

12
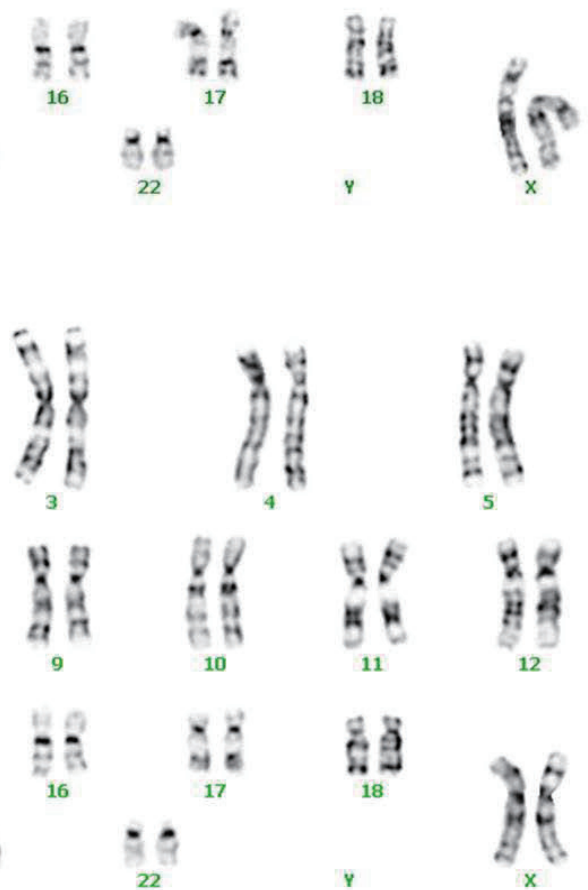

Fig. 3. The results of a G-banded karyogram revealed A: 47, XX, +14 [7] and B: 46, XX [23]. 
uncultured peripheral blood may more precisely detecting the true level of mosaicism than karyotyping based on selectively cultured T lymphocytes. ${ }^{19)}$ Several reports reveal that G-banded karyotyping may underestimate the level of aneuploidy. ${ }^{3,4,19)}$ Although estimated mosaicism proportion (18.0\%) byarray CGH was lower than by karyotyping (23.3\%) in this study, direct comparison between percent aneuploidy by karyotype using cultured cells and by array CGH using uncultured cell should be interpreted cautiously. Because we only detected counted aneuploidy 14 on PHAstimulated cultured T-cells. Therefore further study estimating aneuploidy 14 on uncultured cell, such as FISH on uncultured blood smear, is warranted.

At present, array $\mathrm{CGH}$ is clearly viewed as a useful diagnostic tool for detecting copy number changes. However, array CGH can miss low-level mosaicism based on usual cut-off levels. Consequently, the results of array CGH should be interpreted with caution. Traditional karyotyping with cultured T-lymphocytes is still the most commonly used diagnostic method for chromosome mosaicism. We suggest that array $\mathrm{CGH}$ isstill required to reinforce the information obtained with karyotyping, especially for clinically suspicious patients.

\section{References}

1. lourov IY, Vorsanova SG, Yurov YB. Chromosomal mosaicism goes global. Mol Cytogenet 2008;1:26.

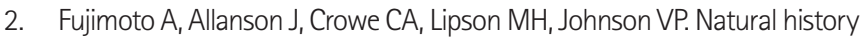
of mosaic trisomy 14 syndrome. Am J Med Genet 1992:44:189-96.

3. Shinawi M, Shao L, Jeng LJ, Shaw CA, Patel A, Bacino C, et al. Low-level mosaicism of trisomy 14: phenotypic and molecular characterization. Am J Med Genet A 2008;146A:1395-405.

4. Cheung SW, Shaw CA, Scott DA, Patel A, Sahoo T, Bacino CA, et al. Microarray-based CGH detects chromosomal mosaicism not revealed by conventional cytogenetics. Am J Med Genet A 2007;143A:1679-86.

5. von Sneidern E, Lacassie Y. Is trisomy 14 mosaic a clinically recognizable syndrome?--case report and review. Am J Med Genet A 2008;146A: 1609-13.
6. Petersen MB, Vejerslev LO, Beck B. Trisomy 14 mosaicism in a 2 year old girl. J Med Genet 1986;23:86-8.

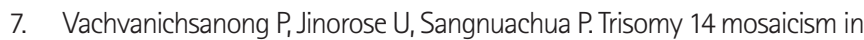
a 5-year-old boy. Am J Med Genet 1991;40:80-3.

8. Lipson MH. Trisomy 14 mosaicism syndrome. Am J Med Genet 1987; 26:541-4.

9. Lynch MF, Fernandes CJ, Shaffer LG, Potocki L. Trisomy 14 mosaicism: A case report and review of the literature. J Perinatol 2004;24:121-3.

10. Johnson VP, Aceto Jr T, Likness C. Trisomy 14 mosaicism: case report and review. Am J Med Genet 1979;3:331-9.

11. Miller DT, Adam MP, Aradhya S, Biesecker LG, Brothman AR, Carter NP, et al. Consensus statement: chromosomal microarray is a first-tier clinical diagnostic test for individuals with developmental disabilities or congenital anomalies. Am J Hum Genet 2010;86:749-64.

12. Ballif BC, Hornor SA, Sulpizio SG, Lloyd RM, Minier SL, Rorem EA, et al. Development of a high-density pericentromeric region $\mathrm{BAC}$ clone set for the detection and characterization of small supernumerary marker chromosomes by array CGH. Genet Med 2007;9:150-62.

13. Moon JS, Lee SY, Nam CM, Choi JM, Choe BK, Seo JW, et al. 2007 Korean National Growth Charts: review of developmental process and an outlook. Korean J Pediatr 2008;51:1-25.

14. Kotzot D. Complex and segmental uniparental disomy updated. J Med Genet 2008;45:545-56.

15. Hur YJ, Hwang T. Complete trisomy 14 mosaicism: first live-born case in Korea. Korean J Pediatr 2012;55:393-6.

16. Ledbetter DH, Engel E. Uniparental disomy in humans: development of an imprinting map and its implications for prenatal diagnosis. Hum Mol Genet 1995;4 Spec No:1757-64.

17. Engel E. A fascination with chromosome rescue in uniparental disomy: Mendelian recessive outlaws and imprinting copyrights infringements. Eur J Hum Genet 2006;14:1158-69.

18. Mattes J, Whitehead B, Liehr T, Wilkinson I, Bear J, Fagan K, et al. Paternal uniparental isodisomy for chromosome 14 with mosaicism for a supernumerary marker chromosome 14. Am J Med Genet A 2007; 143A:2165-71.

19. Ballif BC, Rorem EA, Sundin $K$, Lincicum M, Gaskin S, Coppinger J, et al. Detection of low-level mosaicism by array $\mathrm{CGH}$ in routine diagnostic specimens. Am J Med Genet A 2006;140:2757-67. 